\title{
Pre-supplementation of Crocus sativus Linn (saffron) attenuates inflammatory and lipid peroxidation markers induced by intensive exercise in sedentary women
}

\author{
Mohammad Hosseinzadeh ${ }^{1}$, Hossein TaheriChadorneshin ${ }^{2 *}$, Mahboobe Ajam-Zibad ${ }^{3}$, Seyed-Hosein Abtahi-Eivary ${ }^{4}$ \\ ${ }^{1}$ Department of Sport Sciences, University of Farhangian, Tehran, Islamic Republic of Iran. \\ ${ }^{2}$ Department of Sport Sciences, University of Bojnord, Bojnord, Islamic Republic of Iran. \\ ${ }^{3}$ Exercise Physiology, University of Gonabad, Gonabad, Islamic Republic of Iran. \\ ${ }^{4}$ Department of Clinical Biochemistry, Gonabad University of Medical Sciences, Gonabad, Islamic Republic of Iran.
}

\section{ARTICLE INFO}

Article history:

Received on: 16/03/2017

Accepted on: 30/04/2017

Available online: 30/05/2017

Key words:

Intensive resistance exercise,

Saffron supplementation,

Tumor necrosis factor alpha,

Malondialdehyde, Lactate

dehydrogenase, Creatine

kinase, Inactive women.

\begin{abstract}
Antioxidant and anti-inflammatory actions of Crocus sativus Linn (saffron) in relation to intensive exercise need to be studied. This study aimed to investigate the effect of four weeks of saffron supplementation on serum levels of tumor necrosis factor alpha (TNF- $\alpha$ ), as a marker of inflammation, malondialdehyde (MDA), as a marker of lipid peroxidation, and levels of Lactate dehydrogenase (LDH) and creatine kinase (CK), as two markers of muscle damage, in sedentary women following intensive exercise. After a 4-week period of supplementation $(30 \mathrm{mg} / \mathrm{day})$, the healthy inactive young women performed one bout of acute resistance exercises at $85 \%$ of one-repetition maximum. Using commercial kits, TNF- $\alpha$ and MDA levels were measured using ELISA method. In addition, LDH and CK were measured by photometric method. Data were statistically analyzed by one-way ANOVA $(\mathrm{P}<0.05)$. Our finding showed a significant increases in serum $\mathrm{LDH}(\mathrm{P}=0.015)$, CK $(\mathrm{P}=0.001)$, TNF- $a(\mathrm{P}=0.001)$ and MDA $(\mathrm{P}=0.001)$ levels following one bout of resistance exercise. However, saffron supplementation prevent from increases in LDH ( $\mathrm{P}=0.999), \mathrm{CK}(\mathrm{P}=0.935), \mathrm{TNF}-\mathrm{a}(\mathrm{P}=0.898)$ and MDA ( $\mathrm{P}=0.617)$ levels induced by one bout of resistance exercise. Although, intensive resistance exercise increases muscle damage enzymes, inflammatory and lipid peroxidation markers, however, pre-supplementation of saffron returns these markers to baseline levels. Therefore, it can be concluded that saffron supplementation acts as anti-oxidative and anti-inflammation agent, making it advisable to athletes and coaches involved in intensive resistance exercises.
\end{abstract}

\section{INTRODUCTION}

Resistance exercise has several health promoting affects. Paradoxically, it is also clear that contracting skeletal muscles generate free radicals that results in damage to various body tissues (Powers and Jackson, 2008). Free radicals attack to membrane lipids and eventually led to the production of malondialdehyde (MDA) (Powers and Jackson, 2008; Gadruni et

\footnotetext{
* Corresponding Author

Hossein TaheriChadorneshin, Hossein TaheriChadorneshin, 4 Km of Bojnord-Esfarāyen Highway, University of Bojnord, Bojnord, North Khorasan, Islamic Republic of Iran. Tel: +985832284610, Fax:+985832284610,E-mail: h.taheri@ub.ac.ir
}

al, 2015). Destruction of cells membrane results in releasing of lactate dehydrogenase (LDH) and creatine kinase (CK) into serum (Gadruni et al., 2015; Pareja-Blanco et al., 2016). In this context, an increase in serum MDA, LDH, and CK levels has been reported following one bout of resistance exercise at $70 \%$ of one-repetition maximum (1-RM) (Gadruni et al., 2015; Pareja-Blanco et al., 2016), resistance elastic-band exercise (Gadruni et al., 2015), anaerobic power testing (Hammouda et al., 2012), and runningbased anaerobic sprint tests (Deminice et al., 2013). In addition to oxidative damage, free radical increases expression of tumor necrosis factor alpha (TNF- $\alpha$ ) which in turn results in inflammatory conditions (Fatouros et al., 2010; Townsend et al., 2015; Vincent et al., 2014). 
It has been indicated that one bout of intensive running on treadmill (Yamashita et al., 1999), eccentric resistance exercise (Vincent et al., 2014 and traditional resistance exercise at 70-80\% 1-RM (Fatouros et al., 2010; Townsend et al., 2015) results in serum TNF- $\alpha$ levels. Because of oxidative and inflammatory conditions induced by intensive resistance exercise, athletes and sports researchers sought a way to prevent or reduce these conditions (Altinoz et al., 2016; Hajizadeh Maleki et al., 2016; Dehghan et al., 2016). Athlete's awareness of the benefits of taking different antioxidant supplements has caused a significant rise in the consumption of such substances. On the other hand, the unfavorable results of taking chemical supplements have attracted sport researchers and experts' attention toward herbal supplements (Altinoz et al., 2016; Hajizadeh Maleki et al., 2016; Dehghan et al., 2016; Khazdair et al., 2015).

Saffron (Crocus sativus Linn.) is an herb commonly cultivated in Iran (Khorasan province) and has important health promoting properties (Altinoz et al., 2016; Hajizadeh Maleki et $a l$. , 2016). Recent data from phytochemstry and pharmacological studies have demonstrated that this plant include crocin, crocetin, picrocrocin and a volatile oil component (safranal) (Dehghan et al., 2016; Khazdair et al., 2015). It has been reported that saffron extracts act as anti-atherosclerotic agent through reduction in plasma viscosity and fibrinogen (Ghanbari-Niaki et al., 2015). Moreover, saffron improves diabetic parameters through activating in AMP-activated protein kinase (AMPK)/Glucose transporter type 4 (GLUT4) pathways (Dehghan et al., 2016). In addition, saffron is used in the traditional medicine due to its antioxidant and anti-inflammatory properties (Altinoz et al., 2016). In this context, it has been showed that saffron supplementation reduced MDA and 8-Isoprostane levels and increased superoxide dismutase (SOD) and catalase (CAT) activity in seminal plasma (Hajizadeh Maleki et al., 2016). Also, it has been reported that saffron supplementation reduce MDA levels and xanthine oxidase enzymes (Altinoz et al., 2016). Furthermore, an increase in total antioxidant capacity (TAC) and glutathione levels have been demonstrated following saffron supplementation in diabetic rats (Hosseini et al., 2014).

Considering the aforementioned literature, we hypothesized that saffron extract and its active constituents can attenuate inflammatory and stress oxidative markers induced by intensive exercise. In reality, the effect of saffron supplementation on inflammatory and oxidative damage following intensive resistance exercise is not well elucidated. Therefore, the purpose of present study was to investigate the effect of 4-week saffron supplementation on serum levels of TNF- $\alpha, \mathrm{MDA}, \mathrm{LDH}$ and CK in sedentary women following intensive resistance exercise.

\section{MATERIALS AND METHODS}

\section{Participants}

Forty healthy and sedentary women (19-23 years old) participated in this quasi-experimental study. At first, a health questionnaire was applied to get assured of their health and illness history. Those who had a heart diseases, high blood pressure, diabetes, arthritis, drug addiction or involved in regular physical activity were excluded. Each subject signed a formal letter of consent prior to beginning the investigation. Finally, the subjects were randomly assigned to the four equal groups: control (C), supplementation (S), resistance exercise (RE), and resistance exercise + supplementation $(\mathrm{RE}+\mathrm{S})$ groups. Anthropometric characteristics of subjects are presented in table 1 .

Table 1: Anthropometric and demographic characteristics of subjects in groups.

\begin{tabular}{cccccc}
\hline Groups & $\begin{array}{c}\text { Age } \\
(\text { years })\end{array}$ & $\begin{array}{c}\text { Weight } \\
(\mathbf{k g})\end{array}$ & $\begin{array}{c}\text { Height } \\
(\mathbf{c m})\end{array}$ & $\begin{array}{c}\text { BMI } \\
\left(\mathbf{k g} / \mathbf{m}^{\mathbf{2}}\right)\end{array}$ & WHR \\
\hline $\mathbf{C}$ & $21 \pm 1$ & $55.70 \pm 9$ & $161 \pm 9$ & $21.28 \pm 4$ & $0.94 \pm 0.05$ \\
S & $21 \pm 1$ & $56.40 \pm 6$ & $160 \pm 4$ & $21.50 \pm 4$ & $0.95 \pm 0.31$ \\
RE & $22 \pm 1$ & $56.30 \pm 6$ & $160 \pm 5$ & $22.07 \pm 4$ & $0.98 \pm 0.03$ \\
RE+S & $20 \pm 2$ & $68.60 \pm 12$ & $162 \pm 6$ & $22.04 \pm 3$ & $0.97 \pm 0.06$ \\
\hline
\end{tabular}

Abbreviations: C; Control, S; Supplementation, RE; Resistance exercise, $\mathrm{RE}+\mathrm{S}$; Resistance exercise + supplementation.

\section{Preparing saffron supplement}

The dried saffron stigma was obtained from Bahraman Saffron Co (Mashhad, Iran). In this study, 52 grams of pure stigma (without the white waste) were milled in a porcelain mortar. Then, milled stigmas were steeped by $1800 \mathrm{ml}$ ethanol $(80 \%)$ for 48 hour. The resulting liquid was clear by the filter. Finally, ethanol was dried by evaporation at a temperature of $35-40{ }^{\circ} \mathrm{C}$ (Hajizadeh Maleki et al., 2016; Akhondzadeh et al., 2005). The obtained extract was placed into the capsule. Each capsule contained $30 \mathrm{mg}$ dried extract of saffron. Subjects in the $S$ and $I R+S$ groups received one capsule of saffron extract (30 mg/day, for 4 weeks) (Zibad et al., 2015; Akhondzadeh et al., 2005), whereas the $C$ and RE groups received no supplement. Previous studies have used high dose of saffron supplement (Hajizadeh Maleki et al., 2016; Ghanbari-Niaki et al., 2015). However, other studies have used a dose of $30 \mathrm{mg} /$ day without any side effects (Zibad et al., 2015; Akhondzadeh et al., 2005). Hence, we used this dose for our research.

\section{Nutritional and medicinal control}

At the beginning of the protocol, information on use of medications/supplements was obtained through self-reported questionnaires. Their diet was also taken into account by applying 24-hour dietary recall questionnaire so that meal subjects consisted of $60 \%$ carbohydrate, $30 \%$ fat and $10 \%$ protein. Furthermore, the use of anti-oxidant supplements and drug-taking was controlled over the research period. Subjects were asked to maintain their normal diet during the period of study and consumed a similar high-carbohydrate meal three day before intensive resistance exercise.

\section{Resistance exercise protocol}

At first, subjects in $\mathrm{RE}$ and $\mathrm{RE}+\mathrm{S}$ groups attended in body-building club and one-repetition maximum (1-RM) was 
determined (Zibad et al., 2015; Brzycki, 1993). According to participants, weight was chosen so that the participants lifted it at least once and up to 10 times. Finally, 1-RM determined by Brzycki equation as below (Brzycki, 1993):

$1-\mathrm{RM}=$ Weight $\div[1.0278-(0.0278 \times$ Number of repetitions $)]$

Then, subjects in exercising groups performed one bout of circular resistance exercise at $85 \%$ of 1-RM at 8 stations (bench press, leg press, seated rows, overhead press, seated leg press, arm extension, arm curls and calf raises), each for 3 sets (Zibad et al., 2015). 30 seconds was allocated to the activity rest in each station and 120 seconds for the intervals. At the beginning and end of resistance exercise protocol, warm-up and cool-down were performed (Zibad et al., 2015).

\section{Biochemical estimations}

Twelve-hour fasting blood was taken from antecubital vein before and immediately after resistance exercise (Zibad et al., 2015). The blood samples were put into tubes and centrifuged at $3000 \mathrm{rpm}$ for $10 \mathrm{~min}$ at $4{ }^{\circ} \mathrm{C}$ (Zibad et al., 2015). We used the commercially 96-well ELISA kits to measure the serum contents of TNF- $\alpha$ (Diaclone SAS., France) and MDA (Biocore Diagnostik Ulm, German). The sensitivities of the TNF- $\alpha$ and MDA kits were $8 \mathrm{pg} / \mathrm{ml}$ and $0 / 1 \mu \mathrm{M}$, respectively. Also, serum LDH and CK levels were determined by photometric method (Pars Azmun Co, Karaj, Iran).

The sensitivities of the LDH and CK kits were 5 IU and 4 IU, respectively. The assays were carried out according to the manufacturer's instructions.

\section{Statistical analysis}

Data were analyzed using a commercially available statistics software package (SPSS, version 16.0, Chicago, USA). All data values are presented as means \pm SD for the all groups. Normality and equality of variances were confirmed by ShapiroWilk's and Levene's tests, respectively. Data were analyzed using a one-way ANOVA and Tukey's post-hoc comparison to test the differences between groups in post-exercise stage. The significance level was set at $\mathrm{P}<0.05$.

\section{RESULTS}

The results indicated that the serum MDA levels, as a marker of lipid peroxidation, increased significantly in RE group $(\mathrm{P}=0.001)$. As for TNF- $\alpha$, a pro-inflammatory marker, a significant increase was found in $\mathrm{RE}$ group $(\mathrm{P}=0.001)$. Furthermore, one bout of resistance exercise significantly increased $\mathrm{LDH}(\mathrm{P}=0.015)$ and CK $(\mathrm{P}=0.001)$ levels, as two markers of muscle damage, in sedentary women.

However, saffron supplementation reduced serum MDA $(\mathrm{P}=0.007)$, TNF- $\alpha(\mathrm{P}=0.019)$, LDH $(\mathrm{P}=0.001)$ and $\mathrm{CK}(\mathrm{P}=0.004)$ levels in $\mathrm{S}$ group $(\mathrm{P}=0.007)$. Also, saffron supplementation lowered serum MDA $(\mathrm{P}=0.617), \quad \mathrm{TNF}-\alpha \quad(\mathrm{P}=0.898), \quad \mathrm{LDH}$
$(\mathrm{P}=0.999)$ and $\mathrm{CK}(\mathrm{P}=0.935)$ levels induced by resistance exercise in $\mathrm{RE}+\mathrm{S}$ group.

Table 2: Means and standard deviation of dependent variables in different groups.

\begin{tabular}{cccc}
\hline $\begin{array}{c}\text { Dependent } \\
\text { variables }\end{array}$ & Groups & Pre- exercise & Post- exercise \\
\hline & $\mathrm{C}$ & $44.6 \pm 12.66$ & $46.52 \pm 13.10$ \\
MDA (mol/L $\boldsymbol{\mu})$ & $\mathrm{S}$ & $24.21 \pm 6.52$ & $23.70 \pm 6.48^{*}$ \\
& $\mathrm{RE}$ & $48.61 \pm 1.10$ & $83.5 \pm 18.10 *$ \\
& $\mathrm{RE}+\mathrm{S}$ & $26.12 \pm 5.41$ & $52.00 \pm 14.29$ \\
\hline & $\mathrm{C}$ & $25.40 \pm 8.06$ & $24.8 \pm 13.54$ \\
$\mathbf{T N F}-\boldsymbol{\alpha}(\mathbf{p g} / \mathbf{m l})$ & $\mathrm{S}$ & $10.23 \pm 6.38$ & $9.99 \pm 4.02 *$ \\
& $\mathrm{RE}$ & $29.40 \pm 8.69$ & $63.90 \pm 19.58^{*}$ \\
& $\mathrm{RE}+\mathrm{S}$ & $12.70 \pm 4.67$ & $25.5 \pm 7.26$ \\
\hline & $\mathrm{C}$ & $107.5 \pm 15.23$ & $105.46 \pm 14.3$ \\
& $\mathrm{~S}$ & $56.70 \pm 12.48$ & $58.20 \pm 7.96 *$ \\
$\mathbf{L D H}(\mathbf{U} / \mathbf{L})$ & $\mathrm{RE}$ & $109.9 \pm 16.52$ & $139.20 \pm 40.20 *$ \\
& $\mathrm{RE}+\mathrm{S}$ & $62.61 \pm 10.48$ & $106.60 \pm 6.50$ \\
\hline CK (U/L) & $\mathrm{C}$ & $9.6 \pm 1.26$ & $9.10 \pm 1.15$ \\
\hline & $\mathrm{S}$ & $7.70 \pm 2.48$ & $6.50 \pm 2.06 *$ \\
& $\mathrm{RE}$ & $8.9 \pm 1.14$ & $14.60 \pm 2.67 *$ \\
& $\mathrm{RE}+\mathrm{S}$ & $6.14 \pm 1.97$ & $10.10 \pm 1.97$ \\
\hline
\end{tabular}

The asterisk (*) indicate a significant difference from C group. Abbreviations are the same as are denoted in the legend of Table. 1.

\section{DISCUSSION}

Key factor involving in stress oxidative is intensity of exercise (Powers and Jackson 2008). In reality, resistance exercise increases free radical production through ischemia/reperfusion mechanism (Gadruni et al., 2015; Townsend et al., 2015; CakirAtabek et al., 2010). Cellular integrity disruption induced by lipid peroxidation is thought to result in releasing of $\mathrm{CK}$ and $\mathrm{LDH}$ into serum. In this regard, a significant increase in serum MDA, LDH and $\mathrm{CK}$ was shown in present study that represents lipid peroxidation and muscle damage. In contrast to our findings, no change in serum MDA has reported in active subjects following acute resistance exercise (Cakir-Atabek et al., 2010). Furthermore, Dixon and colleague have revealed that acute resistance exercise (8 circuit resistance exercises, 3 sets of 10 repetitions at 10 repetitions maximum for each exercise) had no significant effect on serum MDA in resistance-trained collegiate men (Dixon et al., 2006). These contradictions in results may be due to different levels of physical fitness of subjects. It has been reported that enhanced antioxidant systems in active subjects scavenge free radicals produced during intensive resistance exercise (CakirAtabek et al., 2010). In addition, Bloomer and coworkers have showed no significant change in serum MDA following 30 minutes of continuous cycling at $70 \%$ of maximal oxygen uptake $\left(\mathrm{VO}_{2} \max \right)$ (Bloomer et al., 2005). Other reasons for this disagreement may be due to the type of performed activity (endurance activity versus resistance activity). Besides, Rosene et al., in a study no change in serum CK and LDH (at 12, 24, and 48 hours post-exercise) have attributed to low number of movements, repetitions and muscle groups (knee extension with $150 \% 1 \mathrm{RM}$ ) (Rosene et al., 2009). Our findings are consistent with another study in terms of the significant increases in serum MDA, LDH 
and CK following short-term maximal exercise (Hammouda et al., 2012), resistance elastic-band exercise (Gadruni et al., 2015), and repeated-sprint exercise (Deminice et al., 2013). The response of these markers following exercise depend on the type and duration of exercise (Gadruni et al., 2015; Hammouda et al., 2012), nutrition and supplementation status (Deminice et al., 2013), and number of movements, repetitions and muscle groups (Gadruni et al., 2015; Pareja-Blanco et al., 2016). Collectively, these factors will determine the amount and the period of their releasing into serum after injury induced by exercise (Koch et al., 2014).

In addition, results of the present study demonstrate that intensive resistance exercise increased serum TNF- $\alpha$. Our finding is consistent with other studies that showed an increase in serum TNF- $\alpha$ following running-based anaerobic sprint test (Deminice $e t$ al., 2013), eccentric resistance exercise (Vincent et al., 2014) and traditional resistance exercise (Fatouros et al., 2010; Townsend et al., 2015). It has revealed that stress oxidative and accumulation of intracellular calcium induced by resistance exercise results in activation of nuclear factor kappa B (NF-kB) (Deminice et al., 2013). In turn, activation of NF- $\mathrm{kB}$ increases expression of TNF- $\alpha$ which subsequently release into the serum (Fatouros et al., 2010; Townsend et al., 2015). In contrast, in two studies have reported that 50 to 60 minutes exercise on ergometer bicycle at moderate intensity (70 to $75 \% \mathrm{VO}_{2} \max$ ) had no significant effect on plasma TNF- $\alpha$ level in healthy young moderately trained men (Brandauer et al., 2013; Ullum et al., 1994). These contradictions in results may be due to differences in type and exercise intensity.

In contrast, our findings showed that saffron supplementation prevent from increases in inflammatory, stress oxidative, and muscle damage markers induced by one bout of intensive resistance exercise. Recently, a reduction in sperm lipid peroxidation markers (MDA and 8-Isoprostane) and DNA fragmentation has been reported following 16-week low-tointensive cycling training and saffron supplementation (Hajizadeh Maleki et al., 2016). Also, xanthine oxidase enzymes and MDA levels increased after swimming exercise, but there was a lower increase in crocin (active constituent of saffron) treated groups (Altinoz et al., 2016). Furthermore, it has been concluded that a combination of saffron supplementation and low-intensity aerobic exercise is effective for improving the TAC levels of diabetic rats (Hosseini et al., 2014).

Another study has pointed out that saffron supplementation through increase in serum paraoxonase/ arylesterase 1 level prevents from peroxidation of lipids in serum following one bout of resistance exercise (Ajam Zibad et al., 2015). It appears that decreased levels of inflammatory and stress oxidative markers following saffron supplementation is due to direct scavenging of free radicals (Hajizadeh Maleki et al., 2016) and increase in antioxidant system (Altinoz et al., 2016; Hajizadeh Maleki et al., 2016).

In this regard, it has been reported that saffron supplementation scavenging reactive oxygen species in seminal plasma (Hajizadeh Maleki et al., 2016). In the context of antioxidant system, it has been shown that saffron (Hajizadeh
Maleki et al., 2016) and crocin (Altinoz et al., 2016) supplementation increased TAC through increases in SOD, CAT activity (Hajizadeh Maleki et al., 2016) and glutathione levels (Altinoz et al., 2016).

\section{CONCLUSION}

Although, intensive resistance exercise increases muscle damage enzymes, inflammatory and lipid peroxidation markers, however, pre-supplementation of saffron returns these markers to baseline levels. Therefore, it can be concluded that saffron supplementation act as anti-oxidative and anti-inflammation agent, making it advisable to athletes and coaches involved in intensive resistance exercises.

\section{ACKNOWLEDGEMENTS}

We thank the participants and the staff of biochemistry laboratory at the Gonabad University of Medical Sciences for their valuable assistance with us in carrying out the biochemical assays.

Financial Support and Sponsorship: Research council of Islamic Azad University, Gonabad branch, Iran, grants this study (number: 93-DP673).

Conflicts of interest: There are no conflicts of interest.

\section{REFRENCESS}

Akhondzadeh S, Tahmacebi-Pour N, Noorbala AA, Amini H, Fallah-Pour H, Jamshidi AH. Crocus sativus L. in the treatment of mild to moderate depression: A double-blind, randomized and placebo-controlled trial. Phytother Res, 2015; 19: 148-151.

Altinoz E, Ozmen T, Oner Z, Elbe H, Erdemli ME, Bag HG. Saffron (its active constituent, crocin) supplementation attenuates lipid peroxidation and protects against tissue injury. Bratisl Lek Listy, 2016; 117: 381-7.

Bloomer RJ, Goldfarb AH, Wideman L, McKenzie MJ, Consitt LA. Effects of acute aerobic and anaerobic exercise on blood markers of oxidative stress. J Strength Cond Res, 2005; 19(2): 276-85.

Brandauer J, Landers-Ramos RQ, Jenkins NT, Spangenburg EE, Hagberg JM, Prior SJ. Effects of prior acute exercise on circulating cytokine concentration responses to a high-fat meal. Physiol Rep, 2013; 1(3), 1-10.

Brzycki M. Strength testing-predicting a one-rep max from reps-to-fatigue. Journal of Physical Education, Recreation \& Dance. 1993; 64(1): 88-90.

Cakir-Atabek H, Demir S, Pinarbasili RD, \& Gunduz N. Effect of different resistance training intensity on indices of oxidative stress. J Strength Cond Res, 2010; 24: 2491-2497.

Dehghan F, Hajiaghaalipour F, Yusof A, Muniandy S, Hosseini $\mathrm{SA}$, Heydari $\mathrm{S}$, et al. Saffron with resistance exercise improves diabetic parameters through the GLUT4/AMPK pathway in-vitro and in-vivo. Sci Rep, 2016; 28: 25139.

Deminice R, Rosa FT, Franco GS, Jordao AA, \& de Freitas EC. Effects of creatine supplementation on oxidative stress and inflammatory markers after repeated-sprint exercise in humans. Nutrition, 2013; 29: 1127-32.

Dixon CB, Robertson RJ, Goss FL, Timmer JM, Nagle EF, Evans RW. The effect of acute resistance exercise on serum malondialdehyde in resistance-trained and untrained collegiate men. J Strength Cond Res, 2006; 20(3): 693-8.

Fatouros I, Chatzinikolaou A, Paltoglou G, Petridou A, Avloniti A, Jamurtas A, et al. Acute resistance exercise results in 
catecholaminergic rather than hypothalamic-pituitary-adrenal axis stimulation during exercise in young men. Stress, 2010; 13: 461-8.

Gadruni K, Mahmmadpour H, \& Gadruni M. Effect of elasticband exercise on muscle damage and inflammatory responses in Taekwondo athletes. Revista Brasileira de Medicina do Esporte, 2015; 21: 297-301.

Ghanbari-Niaki A, Saeidi A, Aliakbari-Beydokhti M, Ardeshiri S, Kolahdouzi S, Chaichi MJ. Effects of circuit resistance training with Crocus sativus (saffron) supplementation on plasma viscosity and fibrinogen. Ann Appl Sport Sci, 2015: 3: 1-10.

Hajizadeh Maleki B, Tartibian B, Mooren FC, Nezhad FY, Yaseri M. Saffron supplementation ameliorates oxidative damage to sperm DNA following a 16-week low-to-intensive cycling training in male road cyclists. J Funct Foods, 2016; 21: 153-166.

Hammouda O, Chtourou H, Chaouachi A, Chahed H, Ferchichi $\mathrm{S}$, Kallel $\mathrm{C}$, et al. Effect of short-term maximal exercise on biochemical markers of muscle damage, total antioxidant status, and homocysteine levels in football players. Asian J Sports Med, 2012; 3: 239-46.

Hosseini SS, Jalili M, Panahi M, Naghilou Z, Dezhahang M. The effect of aerobic exercise and saffron supplementation on antioxidant capacity in diabetic rats. Int J Biosci, 2014; 4: 1-7.

Khazdair MR, Boskabady MH, Hosseini M, Rezaee R, Tsatsakis AM. The effects of Crocus sativus (saffron) and its constituents on nervous system: A review. Avicenna J Phytomed, 2015; 5: 376-391.

Koch AJ, Pereira R, \& Machado M. The creatine kinase response to resistance exercise. J Musculoskelet Neuronal Interact, 2014; 14: 68-77.

Pareja-Blanco F, Rodríguez-Rosell D, Sánchez-Medina L, Ribas-Serna J, López-López C, Mora-Custodio R, et al. Acute and delayed response to resistance exercise leading or not leading to muscle failure. Clin Physiol Funct I, 2016; 36: 1-10.

Powers SK, \& Jackson MJ. Exercise-induced oxidative stress: cellular mechanisms and impact on muscle force production. Physiol Rev, 2008; 88: 1243-76.
Rosene J, Matthews T, Ryan C, Belmore K, Bergsten A, Blaisdell J, et al. Short and longer-term effects of creatine supplementation on exercise induced muscle damage. J Sports Sci Med, 2009; 8(1), 89-96.

Townsend JR, Hoffman JR, Fragala MS, Jajtner AR, Gonzalez AM, Wells AJ, et al. TNF- $\alpha$ and TNFR1 responses to recovery therapies following acute resistance exercise. Front Physiol, 2015; 6: 1-6.

Ullum H, Haahr PM, Diamant M, Palmø J, Halkjaer-Kristensen J, Pedersen BK. Bicycle exercise enhances plasma IL-6 but does not change IL-1 alpha, IL-1 beta, IL-6, or TNF-alpha pre-mRNA in BMNC. J Appl Physiol (1985), 1994; 77(1): 93-97.

Vincent HK, Perciva 1S, Creasy R, Alexis D, Seay AN, Laura Ann Z, et al. Acute effects of enhanced eccentric and concentric resistance exercise on metabolism and inflammation. J Nov Physiother, 2014; 4: 117.

Yamashita N, Hoshida S, Otsu K, Asahi M, Kuzuya T, \& Hori M. Exercise provides direct biphasic cardioprotection via manganese superoxide dismu-tase activation. J Exp Med. 1999; 189: 1699-706.

Zibad MA, Afzalpour ME, Abtahi SH, Saghebjoo M, Zadeh $\mathrm{MH}$. Interactive effect of Saffron extracts and acute resistance exercise on serum Paraoxonase-1 activity and C-reactive protein. Medicina Sportiva: Journal of Romanian Sports Medicine Society, 2015; 11(4): 2682.

\section{How to cite this article:}

TaheriChadorneshin H, Hosseinzadeh M, Ajam-Zibad M, AbtahiEivary SH. Pre-supplementation of Crocus sativus Linn (saffron) attenuates inflammatory and lipid peroxidation markers induced by intensive exercise in sedentary women. J App Pharm Sci, 2017; 7 (05): $147-151$ 